\title{
Successive Interference Cancellation Algorithm in $m$-QAM Nonorthogonal Multicarrier Systems
}

\author{
Daniel Costa Araújo \\ Department of Teleinformatics-(DETI)
Federal University of Ceará-UFC \\ Fortaleza, Ceará, Brazil \\ Email: araujo@gtel.ufc.br
}

\author{
Antônio Macilio P. Lucena \\ National Institute of Space Research-(INPE) \\ Fortaleza University-(UNIFOR) \\ Eusébio,Ceará, Brazil \\ Email: macilio@roen.inpe.br
}

\author{
João César Moura Mota \\ Department of Teleinformatics(DETI) \\ Federal University of Ceará-UFC \\ Fortaleza, Ceará, Brazil \\ Email: mota@gtel.ufc.br
}

\begin{abstract}
This article investigates non-linear and linear receivers to detect $N$ non-orthogonal $m$-QAM signals through AWGN channel: maximum likelihood receiver (ML), minimum mean square error (MMSE), successive interference cancellation (SIC) and a completely new architecture called modified SIC. An analytical development of the project from each one of the proposed architecture is performed. As contribution we present the performance of the modified SIC in terms of bit error rate (BER) which is close to the performance of the ML receiver with less computational complexity. Another contribution is the determination of the minimum distance among non-orthogonal signals according to spectral overlapping considering $N=5$. Finally, the results are discussed as well as our future prospects of research.
\end{abstract}

Keywords-Nonorthogonal carriers, Maximum Likelihood detector, MMSE, Successive interfere Cancellation (SIC), spectral efficiency.

\section{INTRODUCTION}

It is well known from the literature that orthogonality can provide great advantages for the communication systems like high data rates, better spectral efficiency and power reduction just to name a few. For instance, QAM (Quadrature Amplitude Modulation) modulation uses two orthogonal PAM (Pulse Amplitude Modulation) signals doubling the spectral efficiency. Other systems like Orthogonal Frequency Division Multiplexing (OFDM) can also achieve high spectral efficiency and link reliability [1].

OFDM has had great success and has been largely employed on the actual communication systems, specially in mobile communication systems, providing them to accomplish high data rates [1]. Although orthogonal systems have been widely investigated over the last years, nonorthogonal systems have also its importance. Classic OFDM is composed by a rectangular pulsehape and do not have good spectral shape, therefore it is not a robust scheme for time-frequency dispersive channels [2]. Such kind of channel breaks the orthogonality among the subcarriers and their side lobes and main lobes interfere among themselves, dropping down the system performance [3]. There are a set of scenarios where the orthogonality cannot be maintained without severally reduce the data rates. Therefore, the investigation of nonorthogonal signals play an important role on the detection of the transmitted information in such scenarios [3]-[5].

This work was partially supported by CNPq, CAPES and FUNCAP.
The first nonorthogonal system was presented in [2], [3]. The authors show that the transmitted information can only be recovered under condition of $\Delta f \geq 1 / T$, where $\Delta f$ is the spacing frequency separation between two close subcarriers and $T$ denotes the symbol period. Different from those works, in [6], the authors present two nonorthogonal $m$-QAM signals with frequency separation $\Delta f \leq 1 / T$ for an AWGN channel. A maximum likelihood detector is proposed in order to detect the transmitted $m$-QAM symbols in the two nonorthogonal subcarrier. The proposed receiver structure does not have loss of bit error rate (BER) performance compared to the orthogonal case if $1 \geq \Delta f T>0,6$. This means a better spectral efficient compared with system employing two orthogonal subcarriers. The work [7] also shows that is possible to recover the transmitted information under the condition $\Delta f T<1$. In [7], the proposed system is similar to the one presented in [3]. They employ the Weyl-Heisenberg (W-H) function described as $g(t-k T) e^{j 2 \pi l \Delta f t}$, where $g(t)$ is the pulseshape and $l=1$, $2, \ldots$. However, the results show strong constraints for the $g(t)$ design in order to reduce the interference between the subcarriers.

The works [6] and [8] drive us to generalize the ML detector for $N$ non-orthogonal subcarriers and propose further new receiver structure in order to reducing the computation complexity of ML detector. We derive a nonlinear approach based on the successive interference cancellation (SIC) algorithm, which is traditionally employed in MIMO systems to cancel interference between layers [9]. As contribution we proposed a modified version of SIC and compare with the optimum receiver through the BER curve.

This work is organized as follows: in the section II, the system model is presented to reader. In section III, the ML detector for $N m$-QAM nonorthogonal signals is presented. After this, we derive the SIC receiver and the modified SIC receiver in the section IV. In the sections V and VI, we show our results and our conclusions respectively.

\section{System Model}

Consider $N m$-QAM signals that are combined to create $M=m^{N}$ possibles waveforms. The equivalent baseband signal of $i$-th waveform can be written as follows

$$
s_{i}(t)=\sum_{n=0}^{N-1} x_{i, n}(k) e^{j 2 \pi n \Delta f t} p(t-k T)
$$


where $i=1,2, \ldots, M,(k-1) T \leq t \leq k T$ is the time interval of the $k$-th transmitted symbol, $x_{i, n}(k)$ is the $k$-th $m$ QAM sub-symbol from the $n$-th subcarrier being independent identically distributions (i.i.d) $\forall n$ and $k . p(u)$ is a rectangular pulseshape with unitary amplitude and $u \in[0, T]$. The variable $\Delta f$ defines the frequency separation between two subcarrier. Under $\Delta f<1 / T$ condition, there is a frequency overlapping between the main lobes of the subcarriers of $s_{i}(t)$. The received signal in an AWN channel is given by:

$$
r(t)=s_{i}(t)+q(t)
$$

where $q(t)$ is a baseband white Gaussian noise with zero mean and spectral power $N_{o}$. Note that, under the $\Delta f<1 / T$ condition, there is no orthogonality among the subcarriers causing inter-carrier interference (ICI).

\section{MAXIMUM LiKeliHOOd RECEIVER}

The Maximum Likelihood (ML) criterion maximizes the probability density function of the received signal $r(t)$ given $s_{i}(t)$, named as $f\left[r(t) / s_{i}(t)\right]$. This is the optimum criterion for AWGN channels and can be implemented through the minimum distance [10]

$$
D_{k}\left[r(t), s_{i}(t)\right]=\int_{(k-1) T}^{k T}\left|r(t)-s_{i}(t)\right|^{2} d t,
$$

where $i \in[1, M], k \in \mathbb{N}$ and denotes $k T$-th time instant. Another way to implement the ML criterion is performing $M$ correlation metrics $C_{k}\left[r(t), s_{i}(t)\right]$ given by [10]:

$$
\begin{array}{r}
C_{k}\left[r(t), s_{i}(t)\right]=2 \operatorname{Re}\left\{\int_{(k-1) T}^{k T} r(t) s_{i}^{*}(t) d t\right\} \\
-\int_{(k-1) T}^{k T}\left|s_{i}(t)\right|^{2} d t,
\end{array}
$$

where $i=1,2,3, \ldots, M$ and $E_{i}(k)$ is the energy of the transmitted signal $s_{i}(t)$ in the time interval $(k-1) T \leq t \leq k T$ and given by:

$$
\begin{gathered}
E_{i}(k)=\int_{(k-1) T}^{K T}\left|s_{i}(t)\right|^{2} d t=E_{p}\left\{\sum_{n=0}^{N-1}\left|x_{i, n}(k)\right|^{2}+\right. \\
\left.\sum_{n=0}^{N-1} \sum_{\substack{m=0 \\
m \neq n}}^{N-1} x_{i, n}(k) x_{i, m}^{*}(k) h_{n, m}(\Delta f T, k)\right\},
\end{gathered}
$$

where,

$h_{n, m}(\Delta f T, k)=\operatorname{sinc}((n-m) \Delta f T) e^{j 2 \pi(n-m) \Delta f T(k-1 / 2)}$,

and $E_{p}=\int_{0}^{T}|p(t)|^{2} d t$ is the pulseshape energy of $p(t)$. $E_{i}(k)$ is a function that depends on the $m$-QAM symbols, the time instant $k$ and $\Delta f T$. Substituting Eq. (1) in Eq. (4), the correlation metric between $r(t)$ and $s_{i}(t)$ can be rewritten as follows

$$
\begin{aligned}
& C_{k}\left[r(t), s_{i}(t)\right]= \\
& 2 \operatorname{Re}\left\{\sum_{n=0}^{N-1} x_{i, n}^{*}(k) \int_{(k-1) T}^{k T} r(t) e^{-j 2 \pi n \Delta f t} d t\right\}-E_{i}(k) .
\end{aligned}
$$

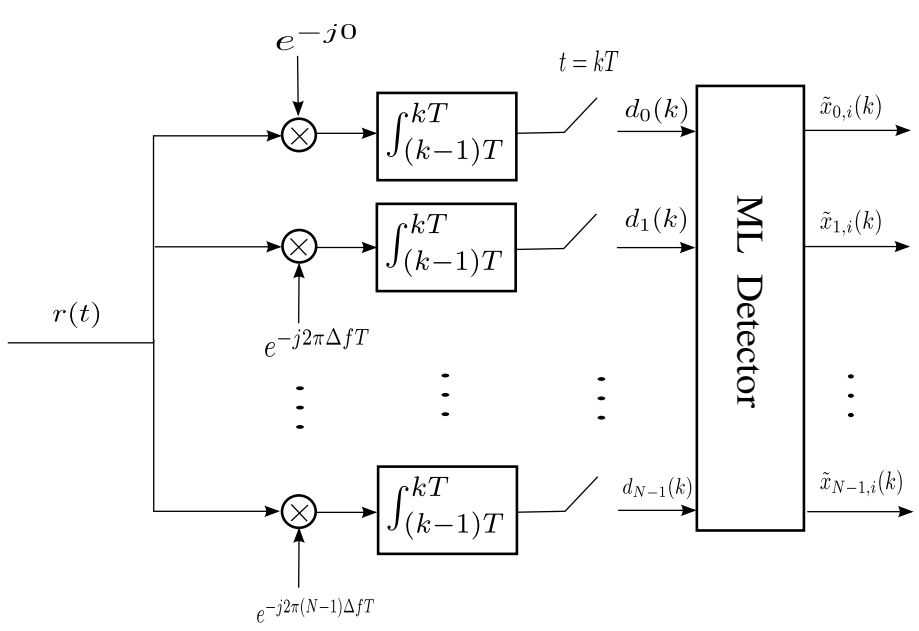

Fig. 1. ML receiver structure for a nonorthogonal multicarrier system.

The ML receiver performs the correlation metric as stated in Eq. (6) for all the possibles transmitted signals and decides the set of $m$--QAM sub-symbols $\left[x_{0, i}(k) x_{1, i}(k), \ldots, x_{N-1, i}(k)\right]$ that maximizes $C_{k}\left[r(t), s_{i}(t)\right]$. Fig. 1 shows the ML receiver structure.

Although ML detector achieves the optimum performance, its computational complexity poses major challenge for NOFDM receiver implementation. By increasing the number of multicarrier the number of the possible transmitted signal grows up according to $M=m^{N}$. For instance, the multicarrier system using a 4-QAM modulation and 16 subcarriers would result in $4^{16}$ different correlation metrics $C_{k}\left[r(t), s_{i}(t)\right]$.

\section{A. Equivalent Discrete Signal Model}

The output signal from the integrator of the $l$-th branch in Fig. 1 can be written as follows

$$
d_{l}(k)=T\left\{x_{l}(k)+\sum_{\substack{n=0 \\ n \neq l}}^{N-1} x_{n}(k) h_{l, n}(\Delta f T, k)\right\}+z_{l}(k)
$$

where $d_{l}(k), x_{j}(k)$, where $j=\{l, n\}$, and $z_{l}(k)=$ $\int_{(k-1) T}^{k T} q(t) e^{j 2 \pi l \Delta f t} d t$ are the output samples of the integrator of the $l$-branch, the $m$-QAM transmitted symbols and the noise in the $l$-th subcarrier at $k$-th time discrete instant, respectively. Note that in Eq. (7), the transmitted symbol in the $l$-th frequency has interference from $(N-1)$ subcarriers and the overlapping is dictated by the value of $\Delta f T$. The smaller $\Delta f T$ is, the larger the interference is.

Eq. (7) shows the information of $l$-th frequency, however, we desire a detector that performs jointly the estimation of all $N m$-QAM transmitted sub-symbols. Writing the set of $N$ equations of (7) as a linear system

$$
\mathbf{d}(k)=T \mathbf{H}(k) \mathbf{x}(k)+\mathbf{z}(k),
$$

where $\mathbf{x}(k)$ is a column vector containing $N$ transmitted 
complex symbols at the time discrete instant $k$,

$$
\mathbf{H}(k)=\left[\begin{array}{ccc}
1 & \ldots & h_{1, N-1}(\Delta f T, k) \\
h_{2,1}(\Delta f T, k) & \ldots & h_{2, N-1}(\Delta f T, k) \\
\vdots & \ddots & \vdots \\
h_{N-1,1}(\Delta f T, k) & \ldots & 1
\end{array}\right],
$$

and $\mathbf{z}(k)$ is the $N \times 1$ complex noise vector. Each entry has zero mean and the correlation matrix of the noise samples among the multicarrier at time instant $k T$ is $E\left[\mathbf{z}(k) \mathbf{z}(k)^{H}\right]=$ $T N_{o} \mathbf{H}(k)$ [11]. If $\Delta f T=1, \mathbf{H}(k)$ becomes a diagonal matrix having no interference among the subcarrier, the noise becomes a white process in the frequency and the problem is reduced to the orthogonal case. If $\Delta f T<1$, the orthogonality condition is broken and $\mathbf{H}(k)$ is cyclostationary.

\section{NONLINEAR RECEIVER}

In order to separate the signals from the nonorthogonal subcarriers at the receiver, interference cancellation detection algorithms should be considered to detect the $m$-QAM transmitted symbols. Basically, the detection is performed setting one subcarrier as the desired, one per turn, and the other ones are treated as interferers. A filter is designed each turn to extract the desired symbol from the desired subcarrier.

\section{A. Successive Interference Cancellation}

In SIC, the subcarriers are detected sequentially as shown in Fig. 2. Initially, the signal $\mathbf{d}(k)$ goes through a filter detector for the subcarrier (we can apply ZF or MMSE criteria), whose output is used to produce a hard estimate of the symbols at this subcarrier, $\hat{x}_{0}(k)$. Then, the contribution of the first subcarrier to the received signal is estimated and cancelled, generating the signal $\mathbf{d}_{1}(k)$. This process is recursive until the last subcarrier is reached. At the $i$-th subcarrier the signal $\mathbf{d}_{i}(k)$, hopefully free from the interference of subcarriers $0, \cdots, i-1$, goes through a filter detector that tries to mitigate the interference from subcarrier $i+1, \cdots, M$. A hard estimate of the symbol at this subcarriers, $\hat{x}_{i}(k)$, is then produced, based on the output of the detector. Then, the contribution of this layer to the "received signal" $\mathbf{d}_{i}(k)$ is estimated and cancelled. This procedure yields a modified received signal given by

$$
\mathbf{d}_{i+1}(k)=\mathbf{d}_{i}(k)-\hat{x}_{i}(k) \mathbf{h}_{i}(k),
$$

where $\mathbf{h}_{i}(k)$ is the $i$-th column of the "interference" matrix $\mathbf{H}(k)$.

In regard to the filter design, it is calculated following a given criterion such as ZF and MMSE [11]. In this paper we consider MMSE criterion, the filter $\mathbf{W}$ is assigned by minimizing the following cost function

$$
J[\mathbf{W}(k)]=E\left\{\|\mathbf{e}(k)\|^{2}\right\}=\left\|\mathbf{x}(k)-\mathbf{W}^{H}(k) \mathbf{d}(k)\right\|^{2} .
$$

Deriving $J[\mathbf{W}(k)]$ with respect to $\mathbf{w}_{n}(k)$

$$
\begin{aligned}
\frac{\partial J[\mathbf{W}(k)]}{\partial \mathbf{w}_{n}(k)} & =-2 E\left\{x_{n}^{*}(k) \mathbf{d}(k)\right\}+2 \mathbf{R}_{d d} \mathbf{w}_{n}(k) \\
\frac{\partial J\left[\mathbf{W}_{o}(k)\right]}{\partial \mathbf{w}_{n}(k)} & =0 \\
\mathbf{w}_{o, l}(k) & =\mathbf{R}_{d d}^{-1} \mathbf{p}_{l}(k)
\end{aligned}
$$

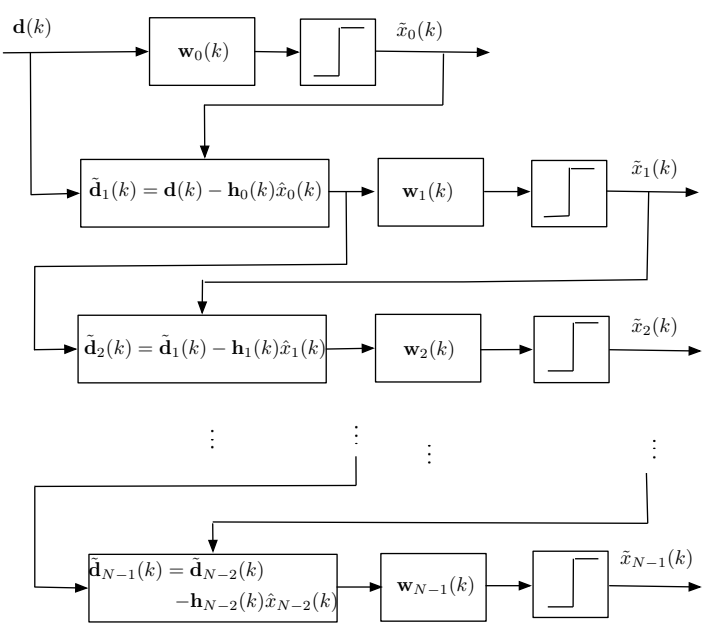

Fig. 2. SIC receiver structure.

where $\mathbf{w}_{o, l}(k)$ is the filter that minimizes the mean square error to the $l$-th subcarrier, $\mathbf{R}_{d d}=E\left\{\mathbf{d}(k) \mathbf{d}(k)^{H}\right\}=$ $\mathbf{H}(k) \mathbf{R}_{x x} \mathbf{H}(k)^{H}+N_{0} T \mathbf{H}(k), \mathbf{R}_{x x}=E\left\{\mathbf{x}(k) \mathbf{x}(k)^{H}\right\}, \mathbf{p}_{l}(k)=$ $E\left\{x_{n}^{*}(k) \mathbf{d}(k)\right\}$ e $E\left\{\mathbf{z}(k) \mathbf{z}(k)^{H}\right\}=N_{0} T \mathbf{H}(k)$. Thus,

$\mathbf{W}_{o}(k)=\left[\begin{array}{lllll}\mathbf{w}_{o, 0}(k) & \mathbf{w}_{o, 1}(k) & \mathbf{w}_{o, 2}(k) & \ldots & \mathbf{w}_{o, N-1}(k)\end{array}\right]$,

represents the optimum transformation matrix that combines linearly.

In this work, we consider $\mathbf{R}_{x x}=\sigma_{x x}^{2} \mathbf{I}, \sigma_{x x}^{2}$ is the power associated to $m$-QAM symbols, $\mathbf{I}$ is the identity matrix, $\mathbf{H}(k)^{H}=\mathbf{H}(k)$, therefore $\mathbf{R}_{d d}=\sigma_{x x}^{2} \mathbf{H}(k) \mathbf{H}(k)+N_{0} T \mathbf{H}(k)$. The vector $\mathbf{p}_{l}(k)$ is given by:

$$
\mathbf{p}_{l}(k)=\left[\begin{array}{lll}
E\left\{x_{l}^{*}(k) d_{1}(k)\right\} & \ldots & E\left\{x_{l}^{*}(k) d_{N-1}(k)\right\}
\end{array}\right]^{T},
$$

to assign $E\left\{x_{l}^{*}(k) d_{n}(k)\right\}$, consider Eq. (7):

$$
\begin{gathered}
E\left\{x_{l}^{*}(k) d_{n}(k)\right\}=\sum_{m=0}^{N-1} E\left\{x_{l}^{*}(k) x_{m}(k)\right\} h_{n, m}(\Delta f T, k) \\
+E\left\{x_{l}^{*}(k) z_{n}(k)\right\} \\
E\left\{x_{l}^{*}(k) d_{n}(k)\right\}=\sigma_{x x}^{2} h_{n, l}(\Delta f T, k) .
\end{gathered}
$$

Eq. (12) can be rewritten as

$$
\begin{aligned}
& \mathbf{p}_{l}(k)=\sigma_{x x}^{2}\left[h_{0, l}(\Delta f T, k) \quad \ldots \quad h_{l-1, l}(\Delta f T, k) \quad 1\right. \\
& \left.h_{l+1, l}(\Delta f T, k) \quad \ldots \quad h_{N-1, l}(\Delta f T, k)\right]^{T} .
\end{aligned}
$$

Using Eq. (11), $\mathbf{W}_{o}(k)$ is

$$
\mathbf{W}_{o}(k)=\left[\begin{array}{llll}
\mathbf{R}_{d d}^{-1} \mathbf{p}_{0}(k) & \mathbf{R}_{d d}^{-1} \mathbf{p}_{1}(k) & \ldots \mathbf{R}_{d d}^{-1} \mathbf{p}_{N-1}(k)
\end{array}\right],
$$

or in other way

$$
\begin{aligned}
\mathbf{W}_{o}(k) & =\sigma_{x x}^{2} \mathbf{R}_{d d}^{-1} \mathbf{H}(k) \\
\mathbf{W}_{o}(k) & =\sigma_{x x}^{2}\left(\sigma_{x x}^{2}\right)^{-1}\left(\mathbf{H}(k) \mathbf{H}(k)+\frac{N_{0} T}{\sigma_{x x}^{2}} \mathbf{H}(k)\right)^{-1} \mathbf{H}(k) \\
\mathbf{W}_{o}(k) & =\left(\mathbf{H}(k) \mathbf{H}(k)+\frac{T}{S N R} \mathbf{H}(k)\right)^{-1} \mathbf{H}(k)
\end{aligned}
$$


where $S N R=\frac{\sigma_{x x}^{2}}{N o}$ defines the signal-noise ratio.

Unfortunately, only the last subcarrier has total cancellation of the interference since only the last one has the information of the estimative from the others $N-1$ subcarriers [9], [11]. In the next section, we present a novel nonlinear receiver to overcome this problem.

\section{B. Modified Successive Interference Cancellation}

The modified SIC implements two SIC process in parallel. The first SIC algorithm cancels successively the interference from the first subcarrier towards to the last one while the second one cancels from the last subcarrier backward to the first subcarrier. At each iteration two symbols are estimated thus at the $N / 2$-th iteration all the symbols are estimated. Although the number of operation is increased up comparing to the traditional SIC, all the subcarriers experience free intersubcarrier interference.

Basically, the equalization can be divided into two steps. The first one is regarded to the first $N / 2$ iterations, when all the symbols are estimated. The second step fulfil the total cancellation of the interference among all the subcarriers estimating new $m$-QAM sub-symbols without interference.

Fig. 3 shows the structure of the modified SIC. At the $j$-th stage, there are two MMSE filters $\mathbf{w}_{j}(k)$ and $\mathbf{w}_{i}(k)$; their outputs are $\hat{x}_{j}(k)$ and $\hat{x}_{i}(k)$ respectively. Be $N$ an even number, then $i+j=N-1$. After $\hat{x}_{j}(k)$ and $\hat{x}_{i}(k)$ detection, the $\{j+1\}$-th stage is

$$
\tilde{\mathbf{d}}_{j+1}(k)=\tilde{\mathbf{d}}_{j}(k)-\mathbf{h}_{j}(k) \hat{x}_{j}(k)-\mathbf{h}_{i}(k) \hat{x}_{i}(k),
$$

where $0<j \leq N / 2-2$ and $N / 2+1 \leq i \leq N-1, \forall j, i$ $\in \mathbb{N}$. Eq. (14) can be rewritten as

$$
\tilde{\mathbf{d}}_{j+1}(k)=\mathbf{d}(k)-\sum_{m=0}^{j} \mathbf{h}_{m}(k) \hat{x}_{m}(k)-\sum_{n=i}^{N-1} \mathbf{h}_{n}(k) \hat{x}_{n}(k) .
$$

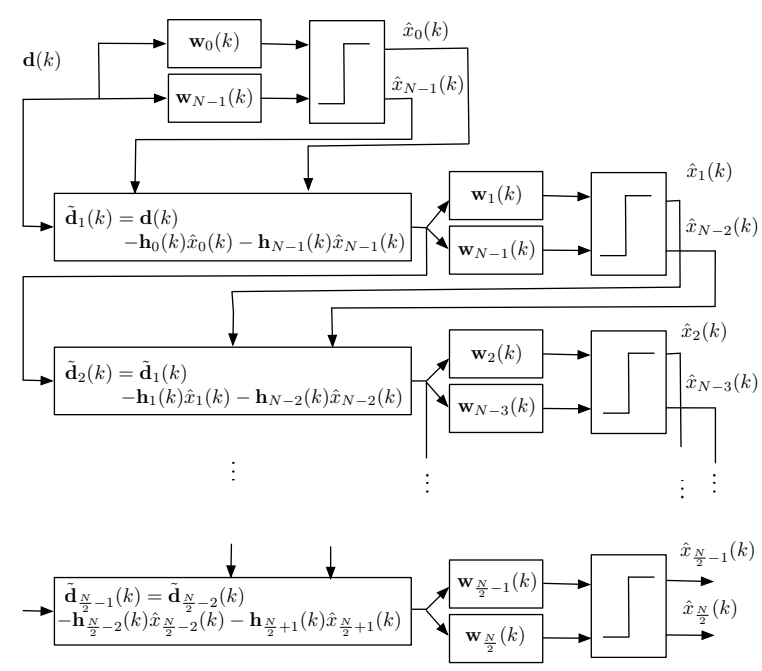

Fig. 3. First phase of modified SIC.

Fig. 4 represents the structure of the second step of the proposed nonlinear equalizer. As in the first stage, there are a
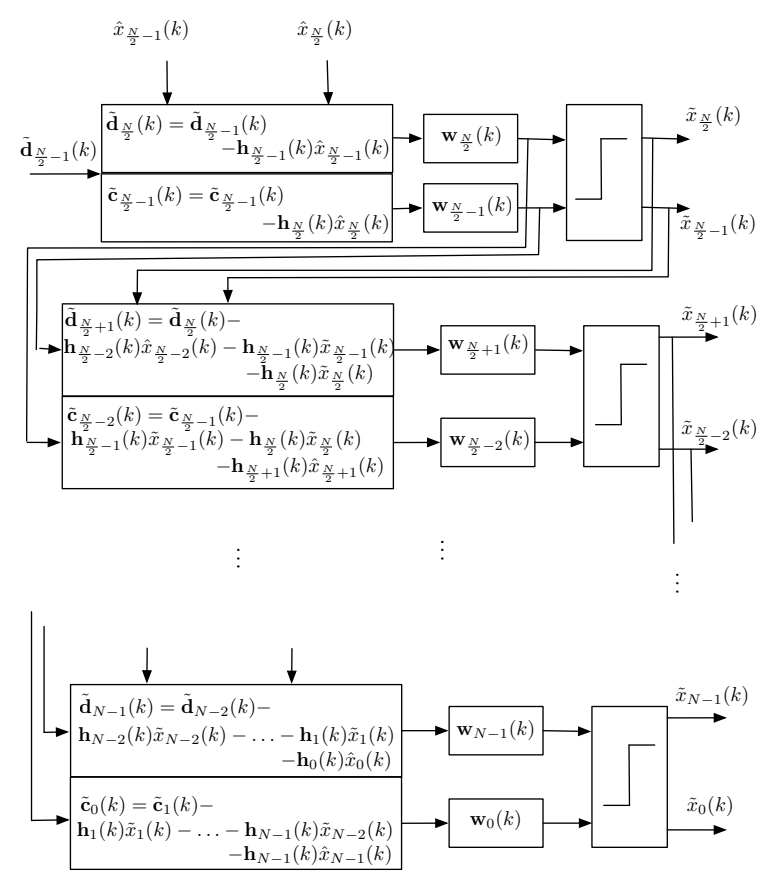

Fig. 4. Second phase of modified SIC.

pair MMSE filter $\mathbf{w}_{j}(k)$ and $\mathbf{w}_{i}(k)$. Their outputs are $\tilde{x}_{j}(k)$ and $\tilde{x}_{i}(k)$, where $0<i \leq N / 2-1, N / 2 \leq j \leq N-1$ and $i+j=N-1$. The signals from $\{j+1\}$-th stage are:

$$
\begin{aligned}
\tilde{\mathbf{d}}_{j+1}(k) & =\tilde{\mathbf{d}}_{j}(k)-\sum_{m=i}^{j} \mathbf{h}_{m}(k) \tilde{x}_{m}(k)-\mathbf{h}_{i-1}(k) \hat{x}_{i-1}(k), \\
\tilde{\mathbf{c}}_{i-1}(k) & =\tilde{\mathbf{c}}_{i}(k)-\sum_{n=i}^{j} \mathbf{h}_{n}(k) \tilde{x}_{n}(k)-\mathbf{h}_{j+1}(k) \hat{x}_{j+1}(k),
\end{aligned}
$$

where $0<i \leq N / 2-2, N / 2+1 \leq j \leq N-1$. For instance, if $i=N / 2-1$ and $j=N / 2$ thus

$$
\begin{aligned}
\tilde{\mathbf{d}}_{N / 2}(k) & =\tilde{\mathbf{d}}_{N / 2-1}(k)-\mathbf{h}_{N / 2-1}(k) \hat{x}_{N / 2-1}(k),(16) \\
\tilde{\mathbf{c}}_{N / 2-1}(k) & =\tilde{\mathbf{c}}_{N / 2-1}(k)-\mathbf{h}_{N / 2}(k) \hat{x}_{N / 2}(k) .
\end{aligned}
$$

\section{RESUlts}

We simulate a nonorthogonal multicarrier system and evaluate it by means of the bit error rate (BER). The receiver structures are MMSE, SIC and modified SIC receiver. We assign the MMSE filter using Eq. (13) and apply on the discrete signal $\mathbf{d}(k)$ [11]

$$
\hat{\mathbf{x}}_{M M S E}(k)=\mathbf{W}_{M M S E}(k) \mathbf{d}(k) .
$$

Furthermore, we compare the minimum distance curve of 2 nonorthogonal multicarriers, presented in [6], with 5 ones and verify if is possible to maintain the same performance of the orthogonal case. We have performed simulations considering 4-QAM modulation.

Fig. 5 shows the minimum distance curve between nonorthogonal symbols and they were simulated employing 2 and 5 subcarriers [12]. Both curves show a range of $\Delta f T$ where the possible symbols of the nonorthogonal multicarrier constellation keep the same minimum distance of the 


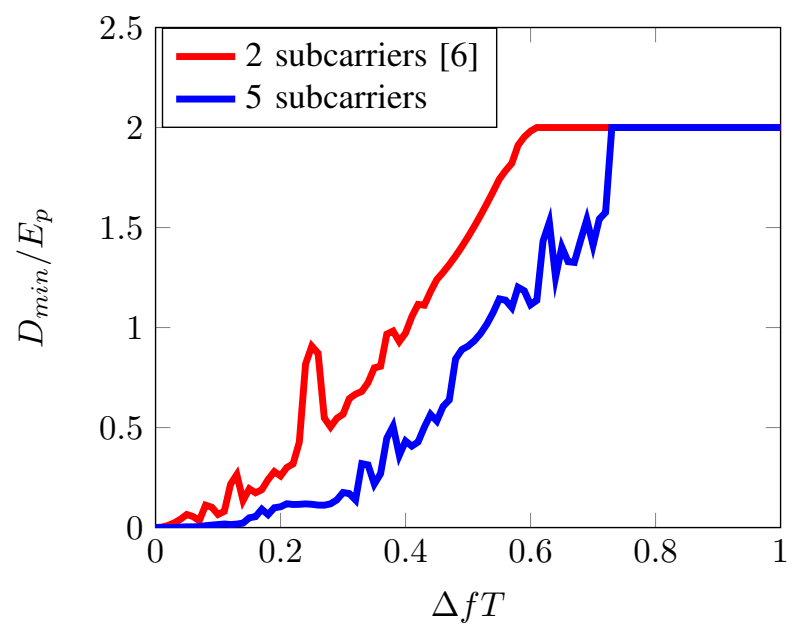

Fig. 5. Minimum distance between 4-QAM symbols with two (red) and five (blue) nonorthogonal subcarriers.

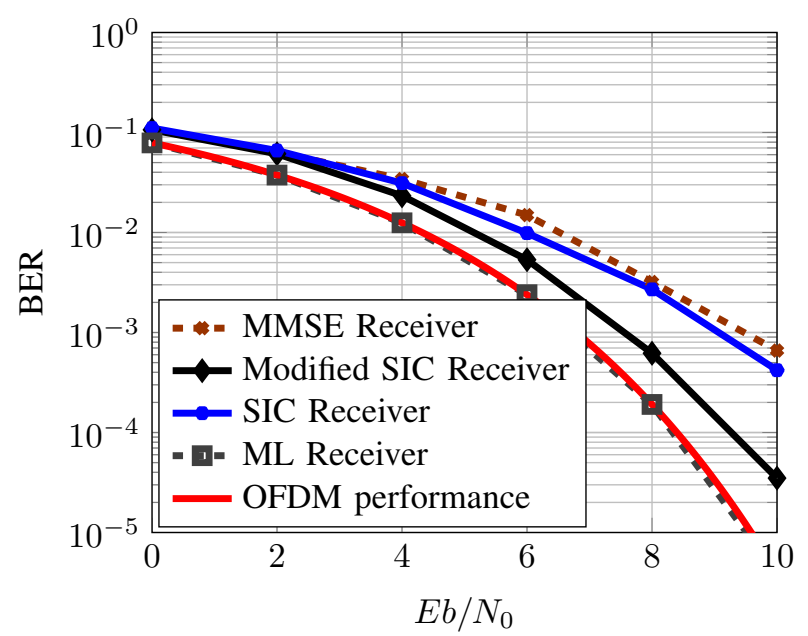

Fig. 6. BER performance of the ML, MMSE, SIC and modified SIC receiver using $\Delta f T=0.73$.

orthogonal case, $\Delta f T=1$. This means that the system can keep the same BER performance in AWGN channel with a better spectral efficiency. On the other hand, the increasing of the number of subcarriers causes a degradation on the BER performance.

Fig. 6 shows the BER performance for the nonorthogonal multicarriers. The simulation was performed with five nonorthogonal subcarriers, $\Delta f T=0.73$ and four different receiver structures: ML, MMSE, SIC and SIC modified. The ML receiver shows no loss of performance comparing to the orthogonal case, this agrees with the minimum distance curve shown in Fig. 5 since the minimum distance between two nonorthogonal symbols with $\Delta f T=0.73$ has the same distance of two OFDM symbols. SIC receiver has slightly better performance than MMSE receiver, less than $0.5 \mathrm{~dB}$ for BER about $10^{-3}$. In regard to the SIC modified, its curve shows that it is a better strategy to reduce the level of interference among the subcarriers in contrast to the SIC and MMSE receiver.

\section{CONCLUSION}

This work investigated the problem of nonorthogonal multicarrier that has already been addressed for two subcarriers in [6] and [8]. We contributed extending for $N$ nonorthognal subcarriers. Furthermore, we evaluate the BER performance using ML, MMSE, SIC and modified SIC receiver. Although without orthogonality, the investigated scheme using the ML receiver can achieve the same BER performance of an OFDM system. It was noticed that the nonorthogonal system can keep the same BER if $0.6 \leq \Delta f T \leq 1$ for $N=2$ and $0.73 \leq$ $\Delta f T \leq 1$ for $N=5$ meaning that nonorthogonal multicarriers can provide an enhanced spectral efficiency. Unfortunately, the ML criterion implies an exponential computational complexity, then we propose a modified SIC receiver that shows a better performance than SIC and MMSE receivers, almost similar to ML receiver, under $\Delta f T=0.73$. Furthermore, the proposed nonlinear receiver has much lower computational complexity than ML. As on going investigation, we intended to study the nonlinear structure in the presence of a dispersive channel.

\section{REFERENCES}

[1] T. Strohmer and S. Beaver, "Optimal OFDM design for time-frequency dispersive channels," IEEE Transactions on Communications, vol. 51, no. 7, pp. 1111-1122, July 2003.

[2] D. Schafhuber, G. Matz, and F. Hlawatsch, "Pulse-shaping OFDM / BFDM systems for time-varying channels: ISI / ICI analysis, optimal pulse design, and efficient implementation," in The 13th IEEE International Symposium on Personal, Indoor and Mobile Radio Communications, vol. 3, Sept 2002, pp. 1012-1016 vol.3.

[3] W. Kozek and A. Molisch, "Nonorthogonal pulseshapes for multicarrier communications in doubly dispersive channels," IEEE Journal on Selected Areas in Communications, vol. 16, no. 8, pp. 1579-1589, Oct 1998.

[4] M. Varanasi and A. Russ, "Noncoherent decorrelative detection for nonorthogonal multipulse modulation over the multiuser gaussian channel," IEEE Transactions on Communications, vol. 46, no. 12, pp. 16751684, Dec 1998.

[5] M. McCloud and L. Scharf, "MMSE multiuser detection for noncoherent non-orthogonal multipulse modulation," in IEEE International Symposium on Information Theory Proceedings., 2000, pp. 356-.

[6] A. M. P. de Lucena, J. C. M. Mota, and C. C. Cavalcante, "Optimum detection of non-orthogonal qam signals with spectral overlapping." IET Communications, vol. 3, no. 2, pp. 249-256, 2009. [Online]. Available: http://dblp.uni-trier.de/db/journals/iet-com/ iet-com3.html\#LucenaMC09

[7] F.-M. Han and X.-D. Zhang, "Wireless multicarrier digital transmission via weyl-heisenberg frames over time-frequency dispersive channels," IEEE Transactions on Communications, vol. 57, no. 6, pp. 1721-1733, June 2009.

[8] A. M. P. Lucena, "Study on communication system with non-orthogonal signals overlapped in frequency," Ph.D. dissertation, Federal University of Ceará-UFC, 2006.

[9] W. Freitas, F. R. P. Cavalcanti, A. de Almeida, and R. Lopes, "Exploiting dimensions of the MIMO wireless channel: multidimensional link adaptation," in 61st IEEE Vehicular Technology Conference VTCSpring, vol. 2, May 2005, pp. 924-928 Vol. 2.

[10] J. Proakis, Digital Communications, ser. McGraw-Hill series in electrical and computer engineering. McGraw-Hill Higher Education, 2001. [Online]. Available: http://books.google.com.br/ books?id=sbr8QwAACAAJ

[11] D. Araújo, "Communications systems with $m$-qam non-orthogonal signals," Master's thesis, Federal University of Ceará-UFC, 2012.

[12] L. F. O. Chamon and A. M. P. Lucena, "Determination of the minimum distance between symbols of the two non-orthogonal m-qam carriers," in XXXI Simpósio Brasileiro de Telecomunicacões, Sept 2013. 